\title{
Global trend of diabetes mortality attributed to vascular complications, $2000-2016$
}

\author{
Wei Ling ${ }^{1,2} \mathbb{0}$, Yi Huang ${ }^{2,3}$, Yan-Mei Huang ${ }^{4}$, Rong-Rong Fan ${ }^{5}$, Yi Sui ${ }^{6^{*}}$ and Hai-Lu Zhao $2,3^{*}$
}

\begin{abstract}
Background: The global epidemic of diabetes mellitus continues to grow and affects developed and developing countries alike. Intensive glycemic control is thought to modify the risks for vascular complications, hence the risks for diabetes-related death. We investigated the trend of diabetic vascular complication-related deaths between 2000 and 2016 in the global diabetes landscape.

Methods: We collected 17 years of death certificates data from 108 countries in the World Health Organization mortality database between 2000 and 2016, with coding for diabetic complications. Crude and age-standardized proportions and rates were calculated. Trend analysis was done with annual average percentage change (AAPC) of rates computed by joinpoint regression.

Results: From 2000 through 2016, 7,108,145 deaths of diabetes were reported in the 108 countries. Among them, $26.8 \%(1,904,787$ cases) were attributed to vascular complications in damaged organs, including the kidneys $(1,355,085$ cases, $71.1 \%)$, peripheral circulatory $(515,293$ cases, $27.1 \%)$, nerves $(28,697$ cases, $1.5 \%)$ and eyes (5751 cases, $0.3 \%)$. Overall, the age-standardized proportion of vascular complication-related mortality was 267.8 [95\% confidence interval $(95 \% \mathrm{Cl}), 267.5-268.1$ c cases per 1000 deaths and the rate was 53.6 (95\% Cl 53.5-53.7) cases per 100,000 person-years. Throughout the 17-year period, the overall age-standardized proportions of deaths attributable to vascular complications had increased $37.9 \%$, while the overall age-standardized mortality rates related to vascular complications had increased 30.8\% (AAPC $=1.9 \%[1.4-2.4 \%, p<0.05]$ ). These increases were predominantly driven by a $159.8 \%$ increase in the rate (AAPC $=2.7 \%[1.2-4.3 \%, \mathrm{p}<0.05]$ ) from renal complications. Trends in the rates and AAPC of deaths varied by type of diabetes and of complications, as well as by countries, regions and domestic income.
\end{abstract}

Conclusion: Diabetic vascular complication-related deaths had increased substantially during 2000-2016, mainly driven by the increased mortality of renal complications.

Keywords: Diabetes mellitus, Vascular complication, Diabetic nephropathy, Mortality

\section{Background}

The global prevalence of diabetes mellitus (DM) has reached 463 million in 2019, and the World Health Organization (WHO) estimated that, worldwide, the number of people living with diabetes would increase

\footnotetext{
*Correspondence: suiyi0126@126.com; zhaohailu9@126.com

${ }^{2}$ Center for Diabetic Systems Medicine, Guangxi Key Laboratory of Excellence, Guilin Medical University, Guilin 541100, China

${ }^{6}$ Department of Clinical Nutrition, The First Affiliated Hospital of Sun YatSen University, Guangzhou 510080, China

Full list of author information is available at the end of the article
}

to 700 million by 2045 [1]. Intensive glycemic control has been shown to be beneficial for the management of microvascular complications of diabetes [2, 3]. As a result, the prevalence of these diabetes-related complications might have also changed [4].

Diabetes has been implicated in all-cause mortality, especially in deaths related to cardiovascular and cerebral vascular disease. [5, 6] Additionally, diabetes is often associated with premature deaths from noncommunicable diseases [7] as well as communicable diseases, including SARS [8], MERS, H1N1 $[9,10]$ and the COVID-19 
[11, 12]. In 2016, an estimated 1.6 million deaths were directly caused by diabetes. Another 2.2 million deaths were attributable to high blood glucose in 2012 [13]. Collectively, mortality of diabetes is often determined by prevalence of diabetes, clinical care, self-management behaviors and risk-factor control. Given that clinical guidelines and recommendations regarding some of these factors have been considerably modified during the past two decades, diabetes-related deaths from microvascular complications might have changed due to tight glycemic control. However, the global burden of vascular complication-related mortality remains unknown. Understanding the mortality trends of vascular complication-related events will help evaluate the current clinical hypoglycemic treatments and guide future diabetes management. Therefore, we aimed to investigate the global burden and trends of diabetic deaths due to vascular complications.

\section{Methods}

\section{Data collection}

We identified countries with available data of all-cause diabetes-related deaths from the WHO Mortality DataBase (WDB), which provides the most comprehensive (standardized national) mortality statistics for countries around the world [14]. The data from the WDB are official national statistics in the sense that they have been transmitted to WHO by the competent authorities of the countries concerned. The WDB comprises deaths registered in national vital registration systems, with underlying cause of death as coded by the relevant national authority. Underlying cause of death is defined as "the disease or injury which initiated the train of morbid events leading directly to death, or the circumstances of the accident or violence which produced the fatal injury" in accordance with the rules of the International Classification of Diseases (ICD). Therefore, there is primary cause-specific attribution of the ICD code to mortality in the death certificates. The WDB contains number of deaths by country, year, sex, age group and cause of death as far back from 1950. Data are included only for countries reporting data properly coded according to the ICD. We used ICD-10 (version 2019) with detailed codes (4th character, ICD-104) to identify all diabetes-related deaths (E10-E14 codes), which include deaths from type $1 \mathrm{DM}$ (E10), type 2 DM (E11), malnutrition-related DM (E12), other specified DM (E13) and unspecified DM (E14). The ICD-10 was endorsed in May 1990 by the Forty-Third World Health Assembly. From 1990 to 2017, a total 10 editions of ICD-10 were released by WHO with the latest edition released in 2016 [15]. We compared all ICD-10 editions and found consistent codes for diabetes mellitus and diabetic complications (E10-E14) during this period. For countries with available ICD codes, we stratified the data by diabetic complications. Vascular complications included in this study for analysis were renal complications (diabetic nephropathy, intracapillary glomerulonephrosis, Kimmelstiel-Wilson syndrome), ophthalmic complications (cataract, retinopathy), neurological complications (amyotrophy, autonomic neuropathy, mononeuropathy, polyneuropathy, autonomic) and peripheral circulatory complications (gangrene, peripheral angiopathy, ulcer), Detail ICD-10 codes for diabetes mellitus and vascular complications used in this study were shown in Additional file 1: Appendix S1. Population data were derived from the WHO Mortality Database and the World Population Prospects 2019 [14, 16].

\section{Procedural}

We identified 135 countries from the database that had included diabetes as a cause of death. Among them, 18 countries used ICD-103 and were therefore excluded. We then extracted mortality data of a 17 -year period between 2000 and 2016. Eventually, 108 countries were included for the final analysis (Additional file 1: Appendix S2). Available years of diabetic vascular complicationrelated deaths, total diabetes-related deaths and mid-year population were provided in Additional file 1: Appendix S3-S5.

\section{Statistical analysis}

To investigate the time trends of vascular complicationrelated deaths, we first estimated the crude proportions and rates using the number of deaths reporting any of renal, ophthalmic, neurological and peripheral circulatory complications as the numerator, divided by the number of all-cause diabetes-related deaths. Age-standardized proportions and rates were then calculated using mid-year population. The direct standardization method was performed to calculate age-standardized proportions and rates using the age-specific (0-4 years, 5-9 years, 10-14 years, 95-99 years, $>100$ years) numbers of diabetic deaths and populations. Secondly, we compared crude and age-standardized proportions and rates of each country, taking the overall estimates of all countries as the reference. Data were reported as odds ratios (ORs). We also compared the differences of the proportions and rates by age groups, regions, DM types, and domestic incomes (based on the World Bank classification) [17].

Using the crude and age-standardized proportions and rates, we performed joinpoint regression to investigate the time trends of diabetic vascular complication-related deaths. This approach has been widely used to study time trends in mortality from causes including infectious [18] and non-infectious diseases. [19] Descriptions of joinpoint regression were shown in Additional file 1: Appendix S6. Briefly, the joinpoint analysis identifies the best 
fit for inflexion points ("joinpoints") at which there is a significant change in trends using a series of permutation tests, with Bonferroni adjustment for multiple comparisons. The joinpoint regression is different than other similar models, like piecewise regression because it has the constrain of continuity at the change-point (s) and the choice of the number of joinpoint (s). Single segment trends were expressed as annual percentage changes (APC), while the average annual percentage changes (AAPC) was used to summarize measures of the overall trend. We used the $\mathrm{Z}$ test to assess whether an APC or AAPC was significantly different from zero. Joinpoint regression analysis was performed for the overall estimates, and for subgroups by sex, type of complications, regions and domestic income. Lastly, we also used the calendar year 2000 as the reference to compare trends of ORs in different subgroups.

We used Microsoft Excel 2010 for data extraction, sorting, and cleaning. IBM SPSS Statistics (version 25.0) and Joinpoint Regression Program (version 4.7.0.0) were used for data analysis. Results were reported with absolute number, percentage and 95\% confidence interval (95\% CI). Proportions were reported as per 1000 deaths, and rates were reported in 100,000 person-years. A p value less than 0.05 was considered statistically significant.

\section{Results}

\section{Overall characteristics}

Between 2000 and 2016, a total of 7,108,145 diabetes-related deaths were reported in the 108 selected countries, including $1,904,787$ cases $(26.8 \%)$ that were attributed to vascular complications. By type of diabetes, there were 88,479 cases of T1DM, 687,959 cases of T2DM, 11,466 cases of malnutrition-related DM, 2326 cases of other specified DM and 1,114,557 cases of unspecified DM. Among the 1,904,787 deaths from vascular complications, the largest proportion was attributed to diabetic nephropathy $(1,355,085$ cases, $71.1 \%$ ), followed by peripheral circulatory complications (515,293 cases $27.1 \%)$, diabetic neuropathy $(28,697$ cases, $1.5 \%$ ) and diabetic retinopathy (5751 cases, $0.3 \%$ ).

\section{Proportions of deaths}

Over the 17-year study period, the overall age-standardized proportions of diabetic vascular complicationrelated deaths were 267.8 (95\% CI 267.5-268.1) cases per 1000 deaths. The highest proportion was found in Singapore (759.6 cases per 1000 deaths, 736.8-782.5), while the lowest was in Sri Lanka (10.9 cases per 1000 deaths, 8.7-13.1) (Additional file 1: Appendix S7). Eighty-four countries $(77.8 \%)$ showed an age-standardized proportion higher than 100 per 1000 deaths. Compared to the overall mean, 68 countries (63.0\%) had an OR $<1$, while
$40(37.3 \%)$ countries had an OR $>1$, ranging from 0.05 (0.04-0.06) in Siri Lanka to 3.37 (3.12-3.65) in Singapore (Fig. 1; Additional file 1: Appendix S8). The age-standardized proportions were higher in men (279.6 cases per 1000 deaths, 278.7-279.7) than in women (257.8 cases per 1000 deaths, 257.4-258.3) (Additional file 1: Appendix S9). The proportions of death due to vascular complications were higher in older persons than in younger individuals, the highest age-standardized proportions were found among 45-64 years (311.2 cases per 1000 deaths, 310.5-311.9), while the lowest were found among $<20$ years (78.0 cases per 1000 deaths, 73.4-82.6) (Additional file 1: Appendix S10). These differences were consistent in both men and women.

By region, Asia (412.8 cases per 1000 deaths, 411.8414.5) had higher proportions of death than other regions, followed by North America (287.5 cases per 1000 deaths, 286.9-288.0) and Oceania (262.2 cases per 1000 deaths, 259.3-265.1). The lowest proportions of death were found in Middle East (128 cases per 1000 deaths, 126.5-129.5) and Africa (160.9 cases per 1000 deaths, 158.2-163.8) (Additional file 1: Appendix S11). By type of vascular complications, renal complications had the highest proportions (190.5 cases per 1000 deaths, 190.4-190.9), followed by peripheral circulatory complications (72.5 cases per 1000 deaths, 72.3-72.7), neurological complications ( 4.0 cases per 1000 deaths, 3.9-4.1) and ophthalmic complications (0.81 cases per 1000 deaths, 0.78-0.83) (Table 1). By domestic income, higher income countries had lower proportions of death (high: 242.3 cases per 1000 deaths, 241.8-242.7; upper-middle: 293.8 cases per 1000 deaths, 293.3-294.3) than lower income countries (lower-middle: 311.5 cases per 1000 deaths, 309.6-313.3; low: 427.9 cases per 1000 deaths, 418.5-437.4).

\section{Rates of deaths}

Between 2000 and 2016, the overall age-standardized rates of vascular complication related deaths were 53.6 (53.5-53.7) cases per 100,000 person-years (Additional file 1: Appendix S12). The highest rate was found in Mauritius (496.9 cases per 100,000 person-years, 490.1503.7), while the lowest rate was documented in Siri Lanka (0.45 cases per 100,000 person-years, 0.38-0.52) (Additional file 1: Appendix S7). Eighty-one countries $(80.2 \%)$ had an age-standardized rate less than 100 cases per 1000 deaths. Compared to the overall mean, 67 countries $(66.3 \%)$ had an $\mathrm{OR}<1$, while $34(33.7 \%)$ countries had an OR $>1$, ranging from $0.006(0.005-0.007)$ in Siri Lanka to 7.15 (6.98-7.32) in Mauritius (Fig. 1; Additional file 1: Appendix S8). Vascular complication related death rates were higher in older persons than for their younger counterparts. The highest rate was observed in the age 

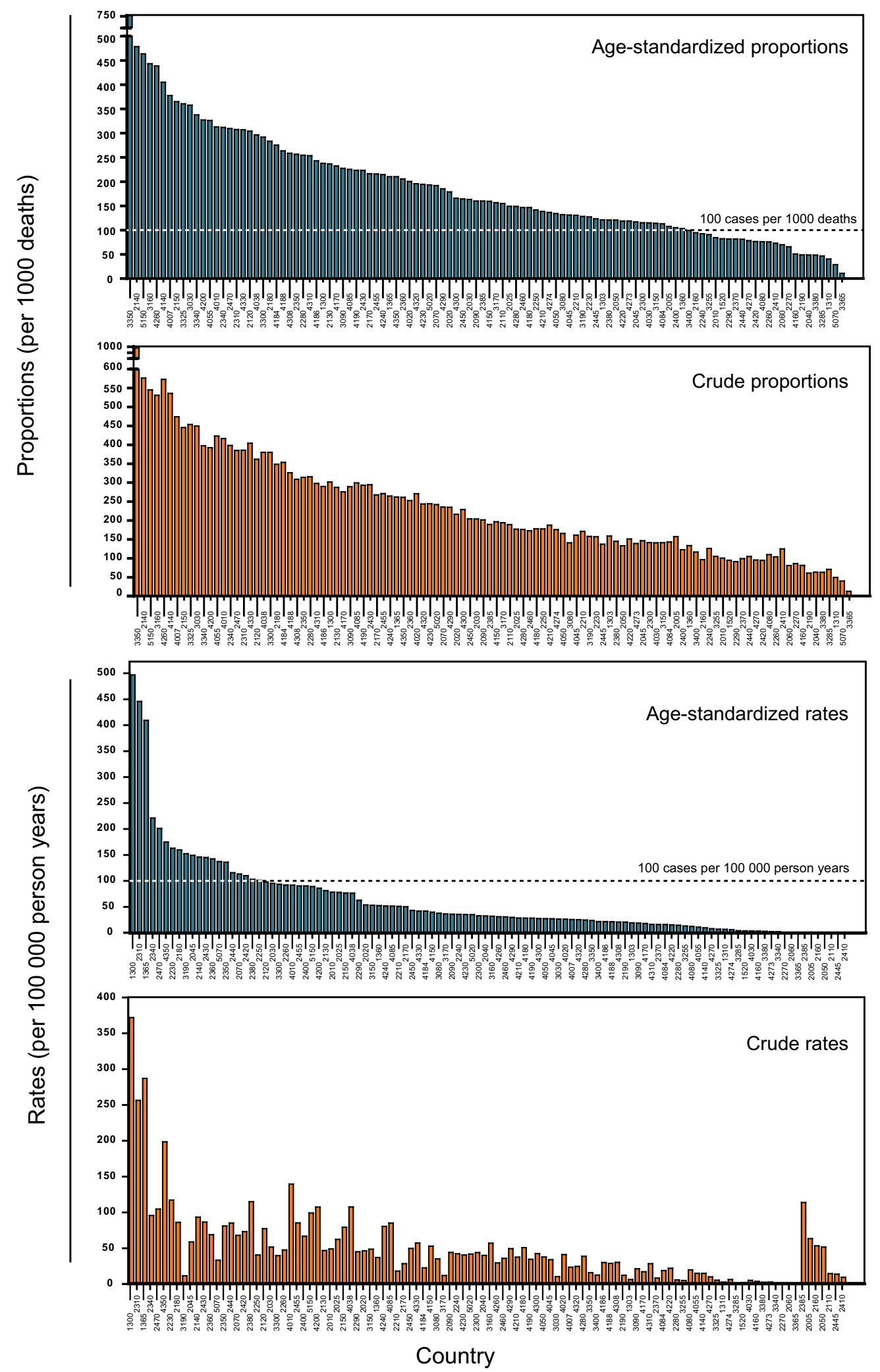

Fig. 1 Crude and age-standardized proportions and rates, by country; Data were sorted by the number of age-standardized proportions and rates; dash line denotes 100 cases per 1000 deaths in proportions and 100 cases per 100000 person years in rates. The number below the $\mathrm{X}$ axis was country codes (corresponding country name was shown in Additional file 1: Appendix S17) 
Table 1 Crude and age-standardized proportions and rates from 2000 to 2016, by vascular complication types

\begin{tabular}{|c|c|c|c|c|c|c|c|c|}
\hline \multirow[t]{2}{*}{ Year } & \multicolumn{4}{|c|}{ Proportions (per 1000 diabetes deaths) } & \multicolumn{4}{|c|}{ Rates (per 100,000 person years) } \\
\hline & Neurological & Ophthalmic & Peripheral circulatory & Renal & Neurological & Ophthalmic & Peripheral circulatory & Renal \\
\hline 2000 & $3.46(3.43)$ & $0.93(0.93)$ & $75.64(77.28)$ & $149.12(143.47)$ & $0.61(0.74)$ & $0.16(0.19)$ & $13.41(14.91)$ & $26.44(29.70)$ \\
\hline 2001 & $3.51(3.48)$ & $0.80(0.80)$ & $82.15(83.32)$ & $149.17(145.38)$ & $0.65(0.77)$ & $0.15(0.17)$ & $15.23(15.85)$ & $27.66(30.69)$ \\
\hline 2002 & $2.96(2.93)$ & $0.75(0.75)$ & $81.28(82.27)$ & $146.57(142.91)$ & $0.59(0.69)$ & $0.15(0.16)$ & $16.21(15.95)$ & $29.23(32.02)$ \\
\hline 2003 & $3.38(3.36)$ & $0.96(0.96)$ & $84.70(84.91)$ & $143.08(141.96)$ & $0.66(0.76)$ & $0.19(0.20)$ & $16.50(15.35)$ & $27.87(30.17)$ \\
\hline 2004 & $3.38(3.38)$ & $0.76(0.76)$ & $81.55(81.58)$ & $141.06(140.45)$ & $0.63(0.72)$ & $0.14(0.15)$ & $15.12(13.79)$ & $26.15(27.98)$ \\
\hline 2005 & $3.60(3.60)$ & $0.74(0.74)$ & 79.38 (79.34) & $142.69(142.55)$ & $0.68(0.78)$ & $0.14(0.15)$ & $15.05(13.70)$ & $27.05(28.60)$ \\
\hline 2006 & $3.60(3.60)$ & $0.69(0.70)$ & $74.94(75.45)$ & $159.61(157.82)$ & $0.73(0.82)$ & $0.14(0.15)$ & $15.23(13.13)$ & $32.43(33.70)$ \\
\hline 2007 & $4.85(4.84)$ & $0.72(0.72)$ & 74.51 (74.68) & $180.51(179.95)$ & $0.97(1.07)$ & $0.14(0.15)$ & $14.96(12.02)$ & $36.24(37.09)$ \\
\hline 2008 & $5.27(5.28)$ & $0.75(0.75)$ & 73.62 (73.19) & $183.77(185.21)$ & $1.02(1.10)$ & $0.15(0.15)$ & $14.26(10.76)$ & $35.59(35.87)$ \\
\hline 2009 & $3.96(3.96)$ & $0.88(0.88)$ & 70.80 (70.96) & $181.57(181.09)$ & $0.84(0.90)$ & $0.19(0.19)$ & $15.11(11.35)$ & $38.76(38.48)$ \\
\hline 2010 & $3.91(3.91)$ & $0.98(0.98)$ & $69.34(69.32)$ & 188.77 (189.09) & 0.85 (0.89) & $0.21(0.21)$ & $14.99(11.21)$ & 40.79 (39.91) \\
\hline 2011 & 3.79 (3.79) & $0.84(0.84)$ & $65.78(65.67)$ & $219.04(219.51)$ & $0.83(0.85)$ & $0.18(0.18)$ & $14.36(10.02)$ & $47.81(45.96)$ \\
\hline 2012 & $4.00(3.99)$ & $0.97(0.97)$ & $64.23(63.69)$ & $224.31(226.22)$ & $0.84(0.85)$ & $0.20(0.19)$ & $13.52(8.82)$ & $47.23(44.62)$ \\
\hline 2013 & $4.67(4.65)$ & $0.92(0.92)$ & $66.39(65.75)$ & $241.43(243.45)$ & $0.99(0.98)$ & $0.20(0.18)$ & $14.03(8.72)$ & $51.03(47.40)$ \\
\hline 2014 & $4.73(4.70)$ & $0.64(0.64)$ & $69.05(68.50)$ & $237.27(238.67)$ & $1.01(0.98)$ & $0.14(0.12)$ & $14.69(8.93)$ & $50.46(46.08)$ \\
\hline 2015 & $4.71(4.69)$ & $0.75(0.74)$ & $67.18(66.39)$ & 244.05 (246.44) & $1.01(0.97)$ & $0.16(0.14)$ & $14.36(8.64)$ & 52.15 (46.89) \\
\hline 2016 & $4.30(4.29)$ & $0.67(0.67)$ & $61.80(61.52)$ & $249.17(250.88)$ & $0.93(0.88)$ & $0.14(0.13)$ & $13.33(7.80)$ & $53.76(47.45)$ \\
\hline Overall & $4.04(4.04)$ & $0.81(0.81)$ & 72.49 (72.53) & $190.64(190.45)$ & $0.82(0.87)$ & $0.16(0.16)$ & $14.71(11.71)$ & 38.68 (38.08) \\
\hline
\end{tabular}

Data (crude [age-standardized]) were sorted by year and complication types; proportions (per 1000 diabetes deaths); rates (per 100,000 population years)

group over 65 years (325.81 cases per 100,000 personyears, 325.4-326.3), while the lowest was found in persons of age $<20$ years $(0.10$ cases per 100,000 personyears, 0.09-0.11) (Additional file 1: Appendix S13).

By region, North America had the highest age-standardized death rate due to vascular complications (94.7 cases per 100,000 person-years, 94.5-95.0), followed by South America (66.0 cases per 100,000 person-years, 65.7-66.2) and Oceania (50.8 cases per 100,000 personyears, 50.1-51.4). By contrast, the Middle East (11.2 cases per 100,000 person-years, 11.1-11.4) and Africa (13.0 cases per 100,000 person-years, $12.7-13.2$ ) had the lowest rates (Additional file 1: Appendix S14). By complication type, the highest rate was found in renal complications (38.1 cases per 100,000 person-years, 38.0-38.2), followed by peripheral circulatory complications (11.7 cases per 100,000 person-years, 11.6-11.8), neurological complications (0.87 cases per 100,000 person-years, $0.86-0.88)$, and ophthalmic complications ( 0.16 cases per $100,000$ person-years, $0.15-0.17$ ) (Table 1$)$. Higher agestandardized death rates were observed in higher income countries (high: 46.6 cases per 100,000 person-years, 46.5-46.7; upper-middle: 75.7 cases per 100,000 personyears, 75.6-75.8) than in the other groups (lower-middle: 27.6 cases per 100,000 person-years, 27.4-27.8; low: 12.9 cases per 100,000 person-years, 12.5-13.3).
Trend of diabetic death rates due to vascular complications From 2000 to 2016, overall rates of deaths due to diabetes vascular complications had increased $30.8 \%$, from 46.0 cases per 100,000 person-years $(45.7-46.3)$ to 60.2 cases (59.7-60.3) per 100,000 person-years. Compared to the rates in the year 2000, the age-standardized OR increased from 1.06 (1.05-1.07) in 2001 to 1.30 (1.29$1.31)$ in 2016, with highest OR occurred in 2013 (1.33, 1.32-1.35) (Fig. 2). The trend of OR also varied by different subgroups (Additional file 1: Appendix S15, S16). The joinpoint model analysis showed that the overall AAPC of rate was $1.9 \%(\mathrm{p}<0.05,1.4 \%-2.4 \%)$. Both men $(\mathrm{AAPC}=2.2 \% ; 1.7 \%-2.7 \% ; \mathrm{p}<0.05)$ and women $(\mathrm{AAPC}=1.6 \% ; 1.1 \%-2.1 \% ; \mathrm{p}<0.05)$ showed an upward trend during the study period. However, the trend varied across different subgroups. As shown in Table 2 and Fig. 3, By DM types, the AAPC was increased in patients with T2DM (AAPC $=7.2 \% ; 6.3 \%-8.2 \%$; $<<0.05$ ) but decreased in persons with T1DM (AAPC $=-8.7 \%$; -14 to $-3.1 \%$; $\mathrm{p}<0.05)$. Similarly, by complication types, the AAPC was higher for diabetic nephropathy $(\mathrm{AAPC}=2.7 \% ; 1.2 \%$ to $4.3 \% ; \mathrm{p}<0.05)$ and diabetic neuropathy $(\mathrm{AAPC}=1.7 \% ; 0.4-2.9 \% ; \mathrm{p}<0.05)$, but lower for diabetic peripheral circulatory complications ( $\mathrm{AAPC}=-4.5 \% ;-5.0$ to $-3.9 \%$; $\mathrm{p}<0.05$ ). By regions, as shown in Fig. 4, trend of annual rate increased in Africa, Caribbean, Europe, Middle East, North America and Oceania, but decreased in Asia and South America. 
Proportions
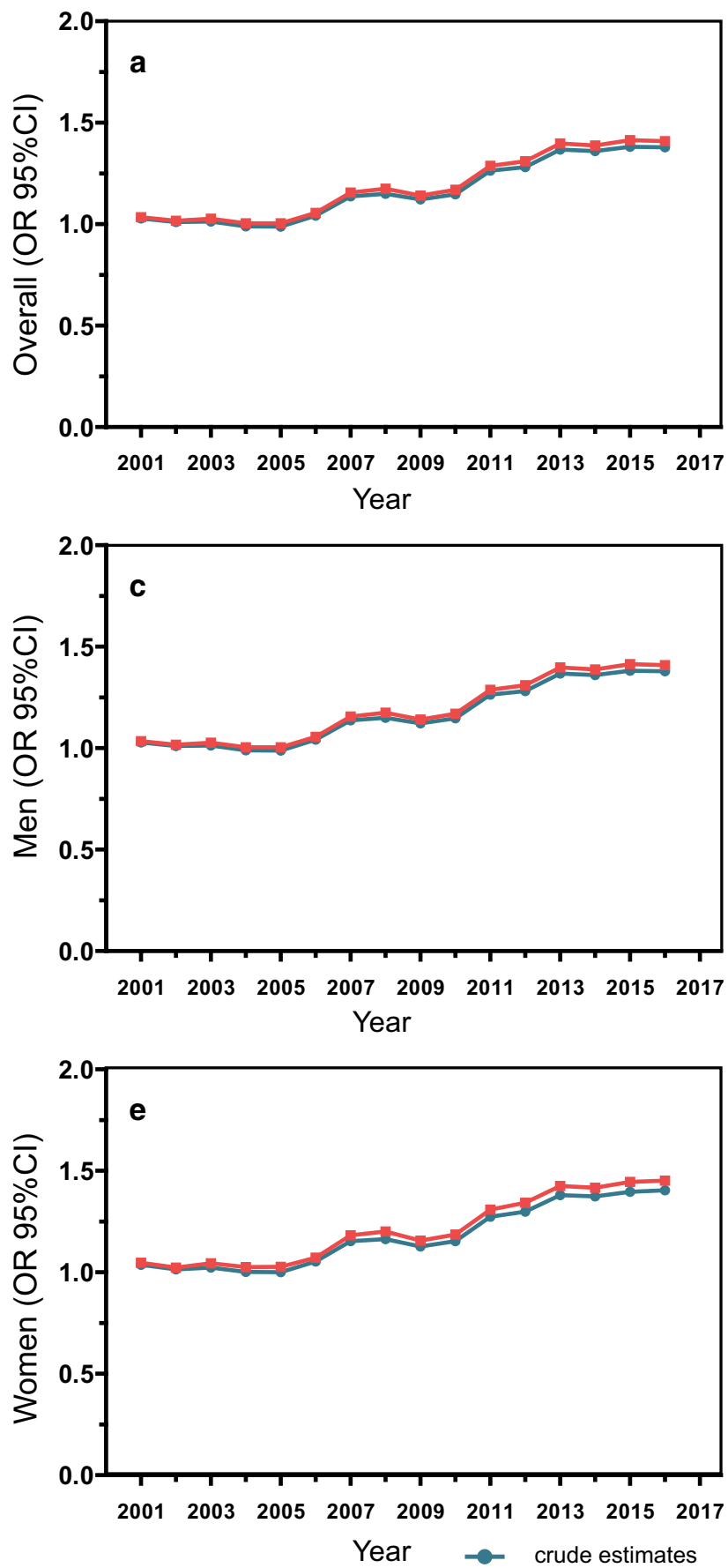

Rates
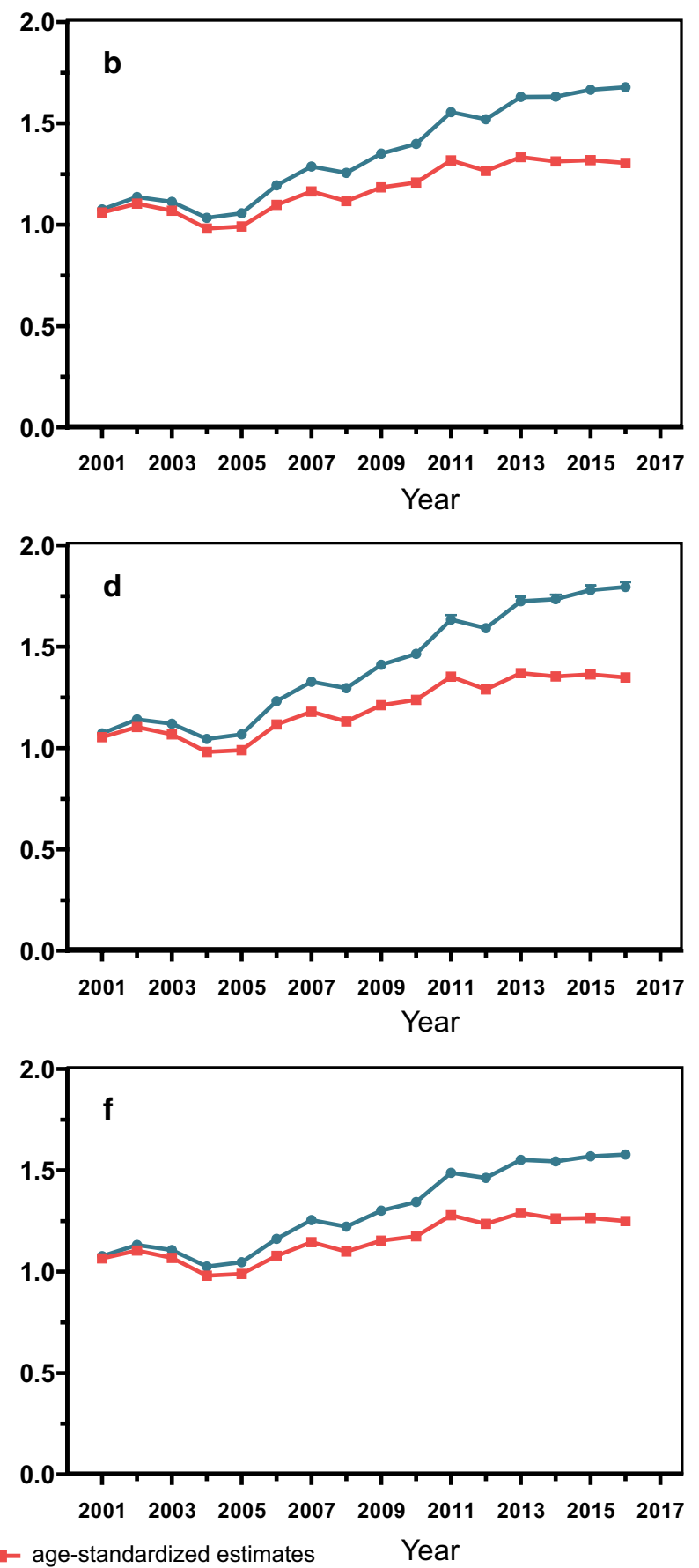

Fig. 2 Odds ratios (ORs) of crude and age-standardized proportions and rates compared to year 2000, by sex; Data presented as estimate \pm 95\% Cl; Circles, crude estimates; Squares, age-standardized estimates; $\mathbf{a}, \mathbf{c}$, e represent proportions; $\mathbf{b}, \mathbf{d}$, $\mathbf{f}$ represent rates; $\mathbf{a}$, b were ORs in overall estimates; $\mathbf{c}, \mathbf{d}$ were ORs in male estimates; e, $\mathbf{f}$ were ORs in female estimates

\section{Discussions}

Main findings

In this study, we reported on the burden of vascular complications in a total of over 7 million diabetic deaths in 108 countries during 2000-2016. The proportions and rates of deaths due to vascular complications varied 
Table 2 Crude and age-standardized average annual percentage change in rates by overall and different subgroups

\begin{tabular}{|c|c|c|c|c|c|c|c|c|}
\hline \multirow[t]{2}{*}{ Subgroup trend } & \multicolumn{4}{|c|}{ Crude rates } & \multicolumn{4}{|c|}{ Age-standardized rates } \\
\hline & AAPC & $95 \% \mathrm{Cl}$ & & $P *$ & AAPC & $95 \% \mathrm{Cl}$ & & $P *$ \\
\hline Total & $3.6^{*}$ & 3.1 & 4.1 & $<0.05$ & $1.9^{*}$ & 1.4 & 2.4 & $<0.05$ \\
\hline \multicolumn{9}{|l|}{ By sex } \\
\hline Male & $4.1^{*}$ & 3.5 & 4.6 & $<0.05$ & $2.2^{*}$ & 1.7 & 2.7 & $<0.05$ \\
\hline Female & $3.2^{*}$ & 2.6 & 3.7 & $<0.05$ & $1.6^{*}$ & 1.1 & 2.1 & $<0.05$ \\
\hline \multicolumn{9}{|l|}{ By DM type } \\
\hline T1DM & 0.8 & -1.4 & 3 & 0.7 & -0.3 & -2.5 & 1.9 & -0.3 \\
\hline $\mathrm{T} 2 \mathrm{DM}$ & $7.2^{*}$ & 6.3 & 8.2 & 14.9 & $7.2^{*}$ & 6.3 & 8.2 & 14.9 \\
\hline Malnutrition-related DM & $-7.6^{*}$ & -12.8 & -2 & -2.6 & $-8.7^{*}$ & -14 & -3.1 & -3 \\
\hline Other specified DM & 1.3 & -5.9 & 9.1 & 0.4 & -0.4 & -7.3 & 7.1 & -0.1 \\
\hline Unspecified DM & 1.1 & -1.8 & 4 & 0.7 & -0.2 & -3 & 2.6 & -0.2 \\
\hline \multicolumn{9}{|l|}{ By complication } \\
\hline Neurological & $3.2^{*}$ & 1.9 & 4.5 & $<0.05$ & $1.7^{*}$ & 0.4 & 2.9 & $<0.05$ \\
\hline Ophthalmic & -0.8 & -7.2 & 6.1 & 0.8 & -2.4 & -8.6 & 4.3 & 0.5 \\
\hline Peripheral circulatory & $<0.05$ & -1.3 & 1.4 & 1 & $-4.5^{*}$ & -5 & -3.9 & $<0.05$ \\
\hline Renal & $4.3^{*}$ & 2.7 & 5.9 & $<0.05$ & $2.7^{*}$ & 1.2 & 4.3 & $<0.05$ \\
\hline \multicolumn{9}{|l|}{ By region } \\
\hline Africa & $23.4^{*}$ & 12.2 & 35.8 & $<0.05$ & $20.7^{*}$ & 10.4 & 31.8 & $<0.05$ \\
\hline Asia & -0.6 & -4.1 & 3 & 0.7 & -2.9 & -6.2 & 0.6 & 0.1 \\
\hline Caribbean & 3.6 & -0.4 & 7.8 & 0.1 & 1.9 & -2.4 & 6.4 & 0.4 \\
\hline Europe & $4.7^{*}$ & 2.6 & 6.8 & $<0.05$ & $3.5^{*}$ & 0.2 & 6.8 & $<0.05$ \\
\hline Middle east & $21.1^{*}$ & 13.2 & 29.6 & $<0.05$ & $18.7^{*}$ & 10.8 & 27.2 & $<0.05$ \\
\hline
\end{tabular}

AAPC means average annual percentage change, and the parametric method was used to calculate $95 \% \mathrm{Cl}$; / denotes no available data

${ }^{*}$ p value $<0.05$

substantially by DM types, complications and geographical areas. Overall, the trend of diabetic mortality due to vascular complications continued to increase from 2000 through 2016.

\section{Increased mortality rate in vascular complications}

Over the last two decades, prevalence of diabetes increased rapidly despite promoted programs of prevention and intervention, [1] As a consequence, the prevalence of diabetic complications may also have changed accordingly. [20] Edward and colleagues demonstrated a downward trend of diabetes-related complications between 1990 and 2010, including end stage renal disease (ESRD) and lower extremity amputation (LEA), which had declined by $29 \%$ and $53 \%$, respectively. [4] Harding et al. summarized the evidence and concluded that, [21] with regard to vascular complications, incidences of LEA [22, 23], diabetic retinopathy [24] and ESRD [4, 25] decreased, while diabetic neuropathy increased during the last few decades. Given these findings, the mortality rate due to diabetic vascular complications seemed to have declined during the same period. However, in this study, we found that the overall trend of diabetes-related deaths due to vascular complications continued to increase between 2000 and 2016. Specifically, an upward trend was observed in renal and neurological complication-related deaths, while a downward trend was observed in peripheral circulatory and ophthalmic complication-related deaths. Additionally, we found that the mortality trend due to vascular complications in T1DM decreased during 2000-2010 but increased during 2010-2013, yet the turning points were not present in the combined analysis. On the other hand, for T2DM, the upward trend of mortality was persistent during 2000-2016. These findings indicated that hard endpoints related to vascular complications in T2DM present a serious challenge for diabetes care and management.

\section{Increased mortality in diabetic nephropathy}

In this study, we observed that the mortality trends varied by type of vascular complications. The overall proportions and rates of deaths due to vascular complications had increased $43.8 \%$ and $30.4 \%$, respectively. However, this increase was primarily driven by growth in the renal complications, which had increased $74.8 \%$ and $59.8 \%$, respectively, during the study period. This finding stressed the fact that diabetic nephropathy 


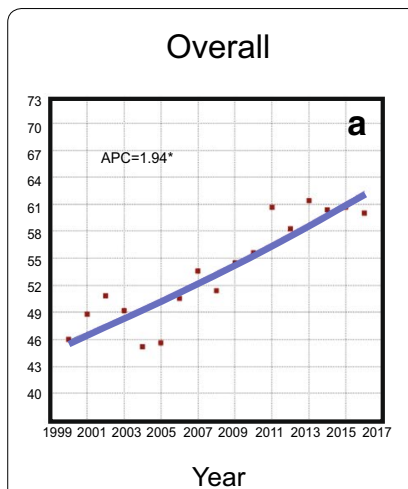

T2 DM

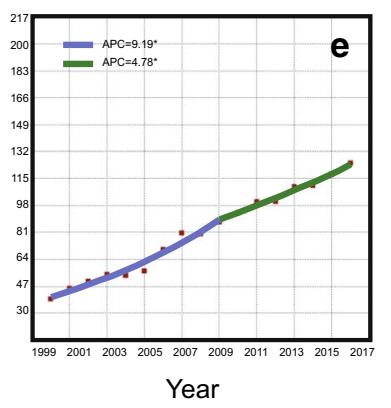

Renal

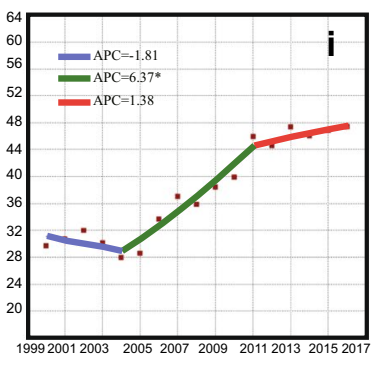

Year
Male

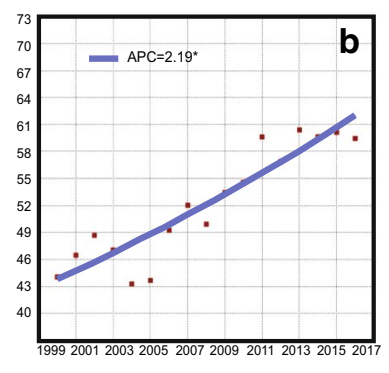

Year

Other specified DM

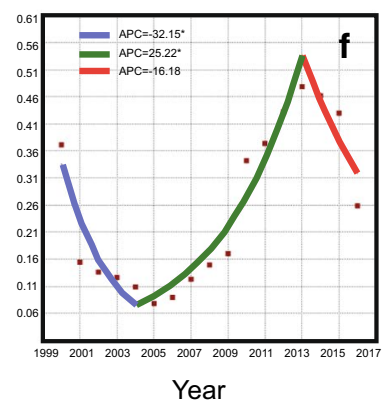

Peripheral circulatory

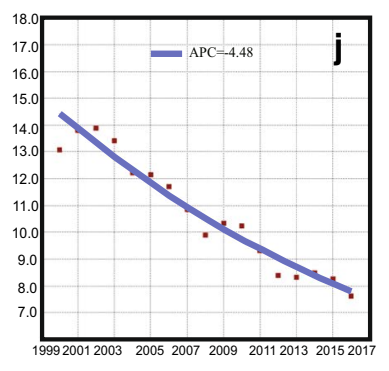

Year
Female

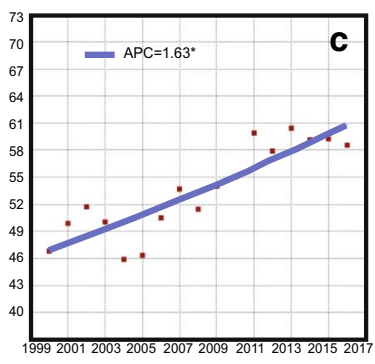

Year

Mulnutrition-related DM

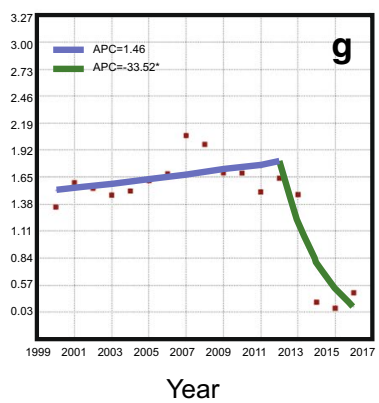

Neurological

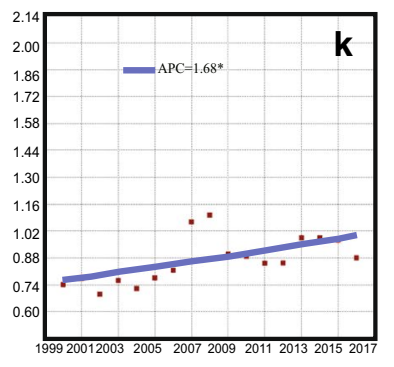

Year
T1 DM

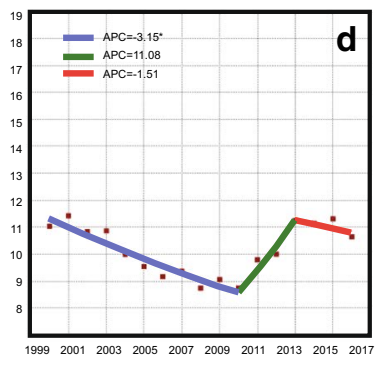

Year

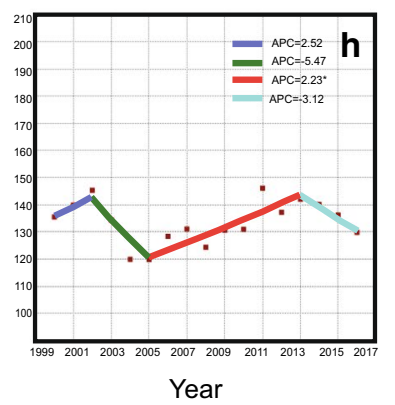

Ophthalmic

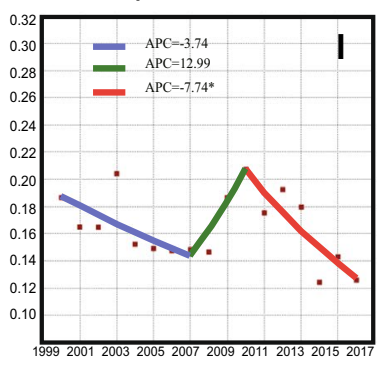

Year

Fig. 3 Trend of age-standardized mortality rates (per 100000 person years) in overall and subgroups by sex, diabetes types and complication types; red squares denote the observed values and line denotes the slope of the APC in different segments; A for overall, B and C for subgroups by sex (B: male, C: female); D-H for subgroups by diabetes types (D: T1DM; E: T2DM; F: other specified DM; G: malnutrition-related DM; H: unspecified DM); I-L for subgroups by complication types (I: renal complication; J: peripheral circulatory complication; K: neurological complication; L: ophthalmic complication); $\mathrm{APC}=$ annual percentage change; * denotes a $p$ value less than 0.05

remains a top priority in the prevention and treatment of diabetes complications. The increased trend of diabetic nephropathy related deaths may partly be explained by the growing prevalence of diabetes. Approximately $40 \%$ of patients with diabetes would develop kidney disease. [26] Worldwide, it is estimated that $80 \%$ of ESRD cases are caused by diabetes or hypertension. [21] Diabetes is the leading cause of ESRD in the United States and the 5-year survival for patients with ESRD is less than 40\%. [27, 28] Additionally, diabetic nephropathy was diagnosed using albuminuria historically. Over the past two decades, non-albuminuric reductions in eGFR are increasingly recognized as diabetic nephropathy. Previously ESRD mortality might have been attributed to hypertension but more recently might have been attributed to diabetic nephropathy. [21, 29] Therefore, the reported increase in diabetic nephropathy related mortality might be affected by a change in understanding of diabetic nephropathy.

Of note, intensive glycemic control has been widely studied for its benefits in renal outcomes [30]. In a 


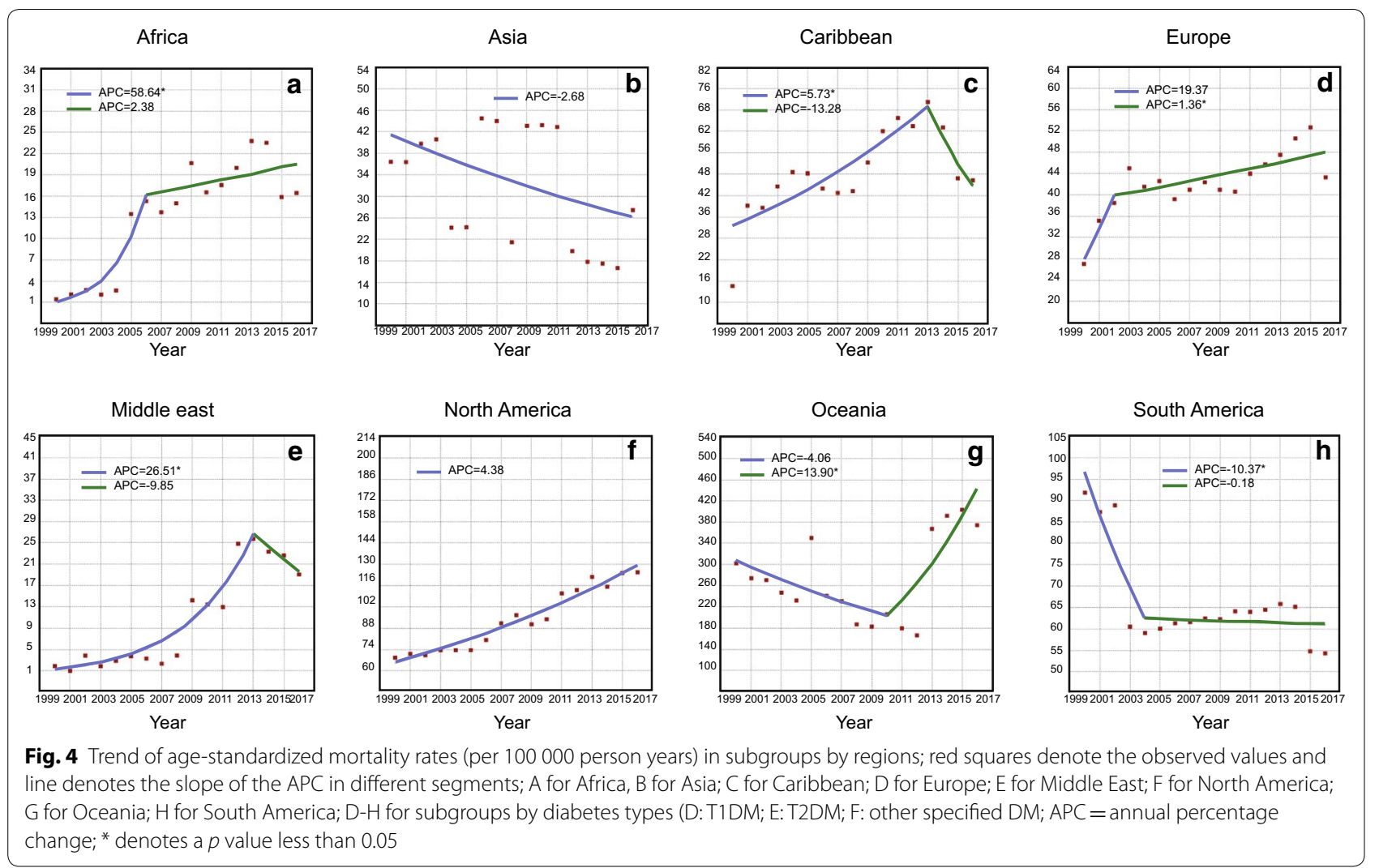

meta-analysis of clinical trials (ACCORD, ADVANCE, UKPDS, and VADT), Zoungas and colleagues found reduced kidney events with intensive glucose control [2]. Another study showed consistent reductions in microalbuminuria surrogates [31]. However, these outcomes do not necessarily translate to downstream improvements in their corresponding hard endpoints $[32,33]$. In contrast, almost all studies have consistently shown a significant increase in severe hypoglycemia and related mortality [34-37]. Correspondingly, CKD is closely correlated with hypoglycemia and may increase the risks of hypoglycemia-induced death and cardiovascular events [38, 39]. Hence, the pros and cons of intensive glycemic control for patients with diabetic nephropathy should be carefully balanced [42].

Differences of mortality rate by geography and time scales This study also demonstrated differences in mortality trends by countries, geographic areas, domestic incomes and time scales. These findings could be explained by pathophysiological differences among individuals of diverse ethnicity, cultural background or variations in diabetes control. Bullock et al. demonstrated that, between 2000 and 2013, the incidence of ESRD with diabetes for American Indian/Alaska Native had declined by $28 \%$, followed by Hispanic (22\%), non-Hispanic white (14\%) and non-Hispanic black people (13\%) in the United States. Yet, during the same period, ESRD incidence remained relatively stable in Asian individuals with diabetes [25]. Additionally, income may also contribute to variable rates of death. A study conducted in England showed that risks of inpatient admission for diabetes increases with socioeconomic deprivation. [40] Reports from IDF have shown that availability of diabetic drugs differs tremendously across income groups. The availability of diabetes supplies ranged from $\sim 80 \%$ in high-income countries to $<15 \%$ in low-income countries. [41] Last, the shifting diagnosis criteria for disease may account for the turning points in some regions. For example, in Australia, a falling trend of diabetic nephropathy was observed between 2000 and 2012 (230 diabetic nephropathy cases in 2012, 21.3\%), whereas this trend was sharply increase from 2013 to 2016 (1240 diabetic nephropathy cases in 2013, 65.9\%). These internal and external inequalities might help explain the differences in the proportions and rates of deaths by regions and countries. 


\section{Co-existing microvascular and macrovascular complications}

The increasing trend of deaths from microvascular complications may correlate with the rising trend of deaths from macrovascular complications [42, 43]. Vascular impairment is closely associated with diabetes. On the one hand, T2DM has a substantial effect on micro- and macrovascular disease and all-cause mortality rates in all age groups, having more pronounced impact on younger individuals [44]. On the other hand, vascular dysfunction may also contribute to the development of T2DM [45]. In patients with T2DM, carotid atherosclerosis parameters predict both cardiovascular and renal outcomes leading to an improved renal risk stratification [46]. An elevated triglyceride-glucose index (TyG index), which is a surrogate of vascular impairment, has been shown positively associated with diabetes prognosis [47] and nephric microvascular damage [48]. Co-existing microvascular and macrovascular complications are frequently observed in patients with diabetes. In this regard, there are considerable difficulties in issuing a death certificate by differentiating microvascular complication from macrovascular complications. The presence and severity of microvascular complications contribute independently to increase the risk of all-cause mortality and cardiovascular events [49]. Therefore, microangiopathy and macroangiopathy may no longer be considered as entirely separate entities but a continuum of systemic vascular damage [50].

\section{Limitations of the study}

To our knowledge, this was the first study to evaluate the mortality trends of diabetic vascular complications in the global landscape. However, there were several limitations. First, we used the WHO mortality database, in which data of some countries with a significant diabetic population, including such as China and India, where accumulate the largest number of people with diabetes mellitus worldwide, are not available. Therefore, the mortality trends of those countries were not reflected in our estimation. Nevertheless, our methodology can be applied to other country-level data for a better understanding of the mortality trend of diabetic vascular complications. Second, the ICD codes used by different countries to identify underlying cause of death may also generate potential bias. In this study, the highest overall age-standardized proportion of diabetic vascular complication-related death was found in Singapore (759.6 cases per 1000 deaths, 95\% CI 736.8-782.5), while the lowest was in Sri Lanka (10.9 cases per 1000 deaths, 95\% CI 8.7-13.1). The difference in mortality might result from the assigned code as unspecified diabetes mellitus without complications (E14.9). The percentage of E14.9-coded deaths was $1.48 \%(16 / 1664)$ in Singapore but 97.64\% (8336/8535) in Sri Lanka. Additionally, patients with diabetes, especially the elderly, often have several complications including hypoglycemia, cardiovascular disease and stroke. Deaths from these complications may be the result of vascular damage but recorded as other causes in different countries. Moreover, LEA is often used as a neurological end point based on the observation that many amputations are a result of diabetes neuropathy and ulcers/infections in the insensate foot. But this is not recorded as a neuropathy end point basing on the ICD rules. Therefore, the comparability and representatives of mortality might be affected across different countries even though the data available from the WBD comprise deaths registered in national vital registration systems and underlying cause of death be coded by the relevant national authority. Third, since certain years of data in some countries were missing, we were not able to analyze trends by country, but estimated the mortality trend with aggregated regional data. Last, estimates of proportions and rates were not based on diabetes prevalence over time as such data were not available. Instead, we used total diabetes deaths, which was indicative of the burden of diabetes.

\section{Conclusions}

In conclusion, we have demonstrated an upward trend of diabetic vascular complications related deaths during the period of 2000-2016. This rising trend of deaths was mainly the result of renal complication in T2DM. Although morbidity of diabetic vascular complications had declined in the past few decades, mortality had continued to climb. This finding indicates an urgent need for developing and implementing effective strategies to reduce the burden of diabetic vascular complications particularly renal damages.

\section{Supplementary information}

Supplementary information accompanies this paper at https://doi. org/10.1186/s12933-020-01159-5.

Additional file 1. Appendix S1: Details of ICD codes for diabetes mellitus and for diabetic vascular complications used in this study. Appendix S2: Flow chart of countries selection. Appendix S3: Available years (grey) for diabetes microvascular complication deaths, by country. Appendix S4: Available years (grey) for total diabetes deaths, by country. Appendix S5 Available years (grey) for midyear population, by country. Appendix S6: Description of joinpoint regression model. Appendix S7: Crude and agestandardized proportions and rates of diabetes microvascular complication related deaths, by country. Appendix S8: Crude and age-standardized odd ratios of proportions and rates compared to overall, by country. Appendix S9: Crude and age-standardized proportions from 2000 to 2016, by sex. Appendix S10: Crude age specific proportions from 2000 
to 2016, by sex. Appendix S11: Crude and age-standardized proportions from 2000 to 2016, by region. Appendix S12: Crude and age-standardized rates from 2000 to 2016, by sex. Appendix S13: Crude age specific rates from 2000 to 2016, by sex. Appendix S14: Crude and age-standardized rates from 2000 to 2016, by region. Appendix S15: Age-standardized odd ratio of rates compared to year 2000, by different subgroups. Appendix S16: Age-standardized odd ratio of proportions compared to year 2000, by different subgroups. Appendix S17: Country code and corresponding country name.

\section{Abbreviations}

T2DM: Type 2 diabetes mellitus; WHO: World Health Organization; ICD: International Classification of Diseases; APC: Annual percentage changes; AAPC Average annual percentage changes; SPSS: Statistical Product and Service Solutions; Cl: Confidence interval; ESRD: End stage renal disease; CKD: Chronic kidney disease; LEA: Lower extremity amputation; TyG: Triglyceride-glucose.

\section{Acknowledgements}

This work was supported by grants from the National Natural Science Foundation of China (81471054, 81660150), the Innovation Project of Guangxi Graduate Education (JGY2015128), and Seeding Fund of the Center for Diabetic Systems Medicine, Guangxi Key Laboratory of Excellence (203030401902). The funders had no role in the study design, data collection and analysis, decision to publish, or preparation of the manuscript.

\section{Authors' contributions}

The conception and design of the study, and draft of this manuscript were performed by LW, SY and ZHL. The generation and data collection were performed by HY, HYM and FRR. The assembly and analysis and/or interpretation of the data were performed by LW and ZHL. All authors read and approved the final manuscript.

\section{Availability of data and materials}

Data of this article were available from the WHO mortality database: https:// www.who.int/healthinfo/statistics/mortality_rawdata/en/.

\section{Ethics approval and consent to participate}

The study protocol was approved by the Ethics Committee Board of Guilin Medical University.

\section{Consent for publication}

Not applicable.

\section{Competing interests}

All the authors declare that they have no conflict of interest.

\section{Author details}

${ }^{1}$ Department of Endocrinology, Xiangya Hospital, Central South University, Changsha 410008, China. ${ }^{2}$ Center for Diabetic Systems Medicine, Guangxi Key Laboratory of Excellence, Guilin Medical University, Guilin 541100, China. ${ }^{3}$ Department of Immunology, Guangxi Area of Excellence, Guilin Medical University, Guilin 541100, China. ${ }^{4}$ Department of Geriatrics, Zhongshan Hospital, Fudan University, Shanghai 200032, China. ${ }^{5}$ Department of Biosciences and Nutrition, Karolinska Institute, 17177 Stockholm, Sweden. ${ }^{6}$ Department of Clinical Nutrition, The First Affiliated Hospital of Sun Yat-Sen University, Guangzhou 510080, China.

Received: 2 August 2020 Accepted: 14 October 2020

Published online: 20 October 2020

\section{References}

1. Saeedi P, Petersohn I, Salpea P, et al. Global and regional diabetes prevalence estimates for 2019 and projections for 2030 and 2045: Results from the International Diabetes Federation Diabetes Atlas, 9 (th) edition. Diabetes Res Clin Pract. 2019;157:107843.

2. Zoungas S, Arima H, Gerstein HC, et al. Effects of intensive glucose control on microvascular outcomes in patients with type 2 diabetes: a meta-analysis of individual participant data from randomised controlled trials. Lancet Diabetes Endocrinol. 2017;5:431-7.

3. Holman RR, Sourij H, Califf RM. Cardiovascular outcome trials of glucose-lowering drugs or strategies in type 2 diabetes. Lancet. 2014;383:2008-17.

4. Gregg EW, Li Y, Wang J, et al. Changes in diabetes-related complications in the United States, 1990-2010. N Engl J Med. 2014;370:1514-23.

5. Raghavan S, Vassy JL, Ho YL, et al. Diabetes mellitus-related all-cause and cardiovascular mortality in a national cohort of adults. J Am Heart Assoc. 2019;8:e011295.

6. An Y, Zhang P, Wang J, et al. Cardiovascular and all-cause mortality over a 23-year period among chinese with newly diagnosed diabetes in the Da Qing IGT and diabetes study. Diabetes Care. 2015;38:1365-71.

7. Rao Kondapally Seshasai S, Kaptoge S, Thompson A, et al. Diabetes mellitus, fasting glucose, and risk of cause-specific death. N Engl J Med. 2011:364:829-41.

8. Yang JK, Feng Y, Yuan MY, et al. Plasma glucose levels and diabetes are independent predictors for mortality and morbidity in patients with SARS. Diabet Med. 2006;23:623-8.

9. Schoen K, Horvat N, Guerreiro NFC, de Castro I, de Giassi KS. Spectrum of clinical and radiographic findings in patients with diagnosis of $\mathrm{H} 1 \mathrm{~N} 1$ and correlation with clinical severity. BMC Infect Dis. 2019;19:964.

10. Wang W, Chen H, Li Q, et al. Fasting plasma glucose is an independent predictor for severity of H1N1 pneumonia. BMC Infect Dis. 2011;11:104.

11. Yang $X, Y u Y, X u J$, et al. Clinical course and outcomes of critically ill patients with SARS-CoV-2 pneumonia in Wuhan, China: a singlecentered, retrospective, observational study. Lancet Respir Med. 2020;8:475-81.

12. Guan WJ, Ni ZY, Hu Y, et al. Clinical characteristics of coronavirus disease 2019 in China. N Engl J Med. 2020;382:1708-20.

13. Global Report on Diabetes. World Health Organization 2016. https://apps. who.int/iris/rest/bitstreams/909883/retrieve. Accessed 2 June 2020.

14. World Health Organization. WHO Mortality Database 2019. https://www. who.int/healthinfo/statistics/mortality_rawdata/en/. Accessed 20 March 2020.

15. Classifications International Classification of Diseases, ICD-10 online versions, Whorld Health Organizations. https://www.who.int/classifications/ icd/icdonlineversions/en/. Accessed 20 Sep 2020.

16. United Nations Department of Economic and Social Affairs Population Dynamics. World Population Prospects. 2019. https://population.un.org/ wpp/. Accessed 25 June 2020.

17. Zhao XY, Xu XX, Yin HS, et al. Clinical characteristics of patients with 2019 coronavirus disease in a non-Wuhan area of Hubei Province, China: a retrospective study. BMC Infect Dis. 2020;20:311.

18. Yang S, Wu J, Ding C, et al. Epidemiological features of and changes in incidence of infectious diseases in China in the first decade after the SARS outbreak: an observational trend study. Lancet Infect Dis. 2017;17:716-25.

19. Boussageon R, Bejan-Angoulvant T, Saadatian-Elahi M, et al. Effect of intensive glucose lowering treatment on all cause mortality, cardiovascular death, and microvascular events in type 2 diabetes: meta-analysis of randomised controlled trials. BMJ. 2011;343:d4169.

20. Gregg EW, Sattar N, Ali MK. The changing face of diabetes complications. Lancet Diabetes Endocrinol. 2016;4:537-47.

21. Harding JL, Pavkov ME, Magliano DJ, Shaw JE, Gregg EW. Global trends in diabetes complications: a review of current evidence. Diabetologia. 2019;62:3-16.

22. Kennon B, Leese GP, Cochrane $L$, et al. Reduced incidence of lowerextremity amputations in people with diabetes in Scotland: a nationwide study. Diabetes Care. 2012;35:2588-90.

23. Narres M, Kvitkina T, Claessen $\mathrm{H}$, et al. Incidence of lower extremity amputations in the diabetic compared with the non-diabetic population: a systematic review. PLoS ONE. 2017;12:e0182081.

24. Wong TY, Mwamburi M, Klein R, et al. Rates of progression in diabetic retinopathy during different time periods: a systematic review and metaanalysis. Diabetes Care. 2009;32:2307-13.

25. Bullock A, Burrows NR, Narva AS, et al. Vital signs: decrease in incidence of diabetes-related end-stage renal disease among American Indians/ Alaska Natives_-United States, 1996-2013. MMWR Morb Mortal Wkly Rep. 2017;66:26-32

26. National KF. KDOQI clinical practice guideline for diabetes and CKD: 2012 update. Am J Kidney Dis. 2012;60:850-86. 
27. de Boer IH, Rue TC, Hall YN, Heagerty PJ, Weiss NS, Himmelfarb J. Temporal trends in the prevalence of diabetic kidney disease in the United States. JAMA. 2011;305:2532-9.

28. United States Renal Data System. USRDS annual data report. https:// www.usrds.org/2018/view/Default.aspx. Accessed 30 May 2020

29. Babaliche P, Nadpara RA, Maldar A. Association between estimated glomerular filtration rate and microvascular complications in type II diabetes mellitus patients: a 1-year cross-sectional study. J Natl Med Assoc. 2019:111:83-7.

30. Perkovic V, Heerspink HL, Chalmers J, et al. Intensive glucose control improves kidney outcomes in patients with type 2 diabetes. Kidney Int. 2013:83:517-23.

31. Duckworth W, Abraira C, Moritz T, et al. Glucose control and vascular complications in veterans with type 2 diabetes. N Engl J Med. 2009;360:129-39.

32. Rodriguez-Gutierrez R, McCoy RG. Measuring what matters in diabetes. JAMA. 2019;321:1865-6.

33. Rodriguez-Gutierrez R, Gonzalez-Gonzalez JG, Zuniga-Hernandez JA, McCoy RG. Benefits and harms of intensive glycemic control in patients with type 2 diabetes. BMJ. 2019;367:15887.

34. Currie CJ, Peters JR, Tynan A, et al. Survival as a function of HbA (1C) in people with type 2 diabetes: a retrospective cohort study. Lancet. 2010;375:481-9.

35. Ray KK, Seshasai SR, Wijesuriya S, et al. Effect of intensive control of glucose on cardiovascular outcomes and death in patients with diabetes mellitus: a meta-analysis of randomised controlled trials. Lancet. 2009:373:1765-72.

36. Zoungas S, Patel A, Chalmers J, et al. Severe hypoglycemia and risks of vascular events and death. N Engl J Med. 2010;363:1410-8.

37. Lee AK, Warren B, Lee CJ, et al. The association of severe hypoglycemia with incident cardiovascular events and mortality in adults with type 2 diabetes. Diabetes Care. 2018;41:104-11.

38. Moen MF, Zhan M, Hsu VD, et al. Frequency of hypoglycemia and its significance in chronic kidney disease. Clin J Am Soc Nephrol. 2009:4:1121-7.

39. Weir MA, Gomes T, Mamdani M, et al. Impaired renal function modifies the risk of severe hypoglycaemia among users of insulin but not glyburide: a population-based nested case-control study. Nephrol Dial Transplant. 2011;26:1888-94.

40. Nishino Y, Gilmour S, Shibuya K. Inequality in diabetes-related hospital admissions in England by socioeconomic deprivation and ethnicity: facility-based cross-sectional analysis. PLoS ONE. 2015;10:e0116689.
41. Access to Medicines and Supplies for People with Diabetes. International Diabetes Federation. https://idf.org/component/attachment s/?task=download\&id=1965. Accessed 17 Apr 2020

42. Roth GA, Forouzanfar MH, Moran AE, et al. Demographic and epidemiologic drivers of global cardiovascular mortality. N Engl J Med. 2015;372:1333-41.

43. Roth GA, Huffman MD, Moran AE, et al. Global and regional patterns in cardiovascular mortality from 1990 to 2013. Circulation. 2015:132:1667-78.

44. Visaria J, Iyer NN, Raval A, et al. Incidence and prevalence of microvascular and macrovascular diseases and all-cause mortality in type 2 diabetes mellitus: a 10-year study in a US commercially insured and medicare advantage population. Clin Ther. 2019;41(1522-36):e1.

45. Muris DM, Houben AJ, Schram MT, Stehouwer CD. Microvascular dysfunction is associated with a higher incidence of type 2 diabetes mellitus: a systematic review and meta-analysis. Arterioscler Thromb Vasc Biol. 2012;32:3082-94

46. Cardoso CRL, Salles GC, Leite NC, Salles GF. Prognostic impact of carotid intima-media thickness and carotid plaques on the development of micro- and macrovascular complications in individuals with type 2 diabetes: the Rio de Janeiro type 2 diabetes cohort study. Cardiovasc Diabetol. 2019;18:2.

47. Zhang M, Wang B, Liu Y, et al. Cumulative increased risk of incident type 2 diabetes mellitus with increasing triglyceride glucose index in normalweight people: the Rural Chinese Cohort Study. Cardiovasc Diabetol. 2017;16:30

48. Zhao S, Yu S, Chi C, et al. Association between macro- and microvascular damage and the triglyceride glucose index in community-dwelling elderly individuals: the Northern Shanghai Study. Cardiovasc Diabetol. 2019;18:95

49. Garofolo M, Gualdani E, Giannarelli R, et al. Microvascular complications burden (nephropathy, retinopathy and peripheral polyneuropathy) affects risk of major vascular events and all-cause mortality in type 1 diabetes: a 10-year follow-up study. Cardiovasc Diabetol. 2019;18:159.

50. Avogaro A, Fadini GP. Microvascular complications in diabetes: A growing concern for cardiologists. Int J Cardiol. 2019;291:29-35.

\section{Publisher's Note}

Springer Nature remains neutral with regard to jurisdictional claims in published maps and institutional affiliations.
Ready to submit your research? Choose BMC and benefit from:

- fast, convenient online submission

- thorough peer review by experienced researchers in your field

- rapid publication on acceptance

- support for research data, including large and complex data types

- gold Open Access which fosters wider collaboration and increased citations

- maximum visibility for your research: over $100 \mathrm{M}$ website views per year

At $\mathrm{BMC}$, research is always in progress.

Learn more biomedcentral.com/submissions 